Rapid Reviews COVID-19

\title{
Review 1: "Impact of B.1.1.7 variant mutations on antibody recognition of linear SARS-CoV-2 epitopes"
}

Debmalya Barh ${ }^{1}$

${ }^{1}$ PI, IIOAB, Genomics, India

Published on: Jun 17, 2021

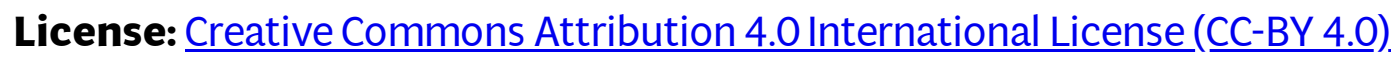




\section{$\underline{\text { RR:C19 Evidence Scale rating by reviewer: }}$}

- Potentially informative. The main claims made are not strongly justified by the methods and data, but may yield some insight. The results and conclusions of the study may resemble those from the hypothetical ideal study, but there is substantial room for doubt. Decision-makers should consider this evidence only with a thorough understanding of its weaknesses, alongside other evidence and theory. Decisionmakers should not consider this actionable, unless the weaknesses are clearly understood and there is other theory and evidence to further support it.

$* * * * * * * * * * * * * * * * * * * * * * * * * * * * * * * * * * * * * * *$

\section{Review:}

In this article, the authors have used high throughput serum epitope repertoire analysis (SERA) and protein-based immunome-wide association studies (PIWAS) to show that the mutations in the B.1.1.7 variant of SARS-CoV-2 do not render existing antibodies to bind to the viral targets. Based on the 579 COVID-19 samples, the authors have shown that the mutations in the spike protein of the B.1.1.7 variant do not have a direct overlap with the epitope hotspots and the only $0.3 \%$ of the cases may show immune evasion due to the del144, N501Y, A570D, and P681H mutations. The D3L mutation in the Nucleoprotein reduces PIWAS signal in 1.5\% of COVID patients however the S235F substantially increases epitope and antigen scores. The mutation in the NS8 shows reduces PIWAS signal in $2.5 \%$ of the COVID patients. However, the NS8 is unlikely to play a role in any immune protection.

The authors have claimed that in the majority of COVID-19 cases, the mutations of the B.1.1.7 strain may not render the antibody responses to the linear epitopes from wild spike glycoprotein and nucleoprotein. However, these findings must be verified with structural analysis and other assays. The study did not consider the COVID-19 vaccinated samples, therefore, the responses of the currently administered vaccines against the B.1.1.7 strain are not known. Therefore, there is no evidence to claim if the currently available vaccines will be ineffective against the B.1.1.7 strain.

It's a preliminary report and authors need to use more samples and also need to demonstrate the efficacy of the currently available wild spike glycoprotein and nucleoprotein-based vaccines against the B.1.1.7 strain with real-time data so that a definite conclusion can be drawn. 
